\title{
Conservative Management of Urinary Stress Incontinence in Selected Post Menopause Patients with Fractional CO2 Laser
}

\author{
JUAN MANUEL TOVAR MD1, MARCO ANTONIO TOVAR MD ${ }^{1}$, ANGGIE SANTILLAN-ROMERO ${ }^{3}$, R. PICHARDO- \\ RODRÍGUEZ MD ${ }^{3}$, MARIO J. VALLADARES-GARRIDO MD MSC $(C)^{2}$, J. ANTONIO GRANDEZ - URBINA MD MSC(C) ${ }^{2}$, H. \\ GARCÍA-PERDOMO MD, MSC, EDD, PHD, FACS 5 \\ ${ }^{1}$ Private Clinic CIRUFEME, Lima, Peru \\ ${ }^{2}$ Universidad Continental, Lima, Peru \\ ${ }^{3}$ Biomedical Research Institute, Universidad Ricardo Palma, Lima, Perú \\ ${ }^{4}$ Medicine School, Universidad del Valle, Cali, Colombia \\ Corresponding author: Juan Manuel Tovar Huamani, informes@cirufeme.com.pe, La Retama Street 321 Los Sauces - Surquillo, Lima- \\ Perú, Telephones: 6624219/+51-992751470
}

\begin{abstract}
Objective: This PUBA study aims to evaluate the effectiveness of the Fractional CO2 Laser in the conservative treatment of Urinary Stress Incontinence in pre-selected Post-Menopausal Patients, with urine loss to Valsalva maneuvers.

Methods: Symptoms of Urinary Stress Incontinence were evaluated before, and 1 month after the last session (3 sessions with a 30-day interval between them) in 50 post-menopausal women (Middle Age - Menopause, Interquartile Range Parity - BMI). Subjective measures (ICIQ SF, VAS) and objective measures such as PAD TEST were used (a dry diaper was previously weighed, and she was instructed to take $500 \mathrm{cc}$ for 15 minutes at rest, and then for 45 minutes to perform Valsalva maneuvers, go up and down stairs, sit, stand up, etc. and then reweigh the diaper) during the study period in order to evaluate the results of the fractional $\mathrm{CO} 2$ laser treatment compared to the baseline.

Results:

Of the 50 patients enrolled, 12 patients in total who presented minimal Urinary symptoms of incontinence (USI) according to the ICIQ questionnaire, all were cured, defining this, as the no loss of urine. Of the 18 patients who presented moderate USI, 10 were cured (55\%), that is, they did not present urine loss, and the other 8 patients (45\%) had partial recovery, but continued losing urine. Of the 20 patients who presented severe USI, none were cured, $9(45 \%)$ of them remained in severity, and 11 (55\%) patients had partial recovery, migrating to the degree of moderate USI.

An objective assessment was also performed using the PAD TEST, of the 12 patients with minimal USI, all were cured, of the 18 patients who presented moderate USI, what was found in the ICIQ was confirmed, with 10 patients cured, and 8 with partial recovery. Likewise, of the 20 patients with severe USI, none were cured.

VAS (Visual Analogue Scale) was also performed at the end of the treatment and after a year by telephone call, to assess the degree of satisfaction, in regards to the minimal USI at the end of the treatment, this was $100 \%$, and after a year it was reduced to $95 \%$. The patients who presented moderate USI at the end of the treatment presented an average degree of satisfaction of $88.8 \%$, and after 1 year it was $72.2 \%$, and in respect of severe USI it was $6.5 \%$ at the end of the laser treatment, and after one year it was $0 \%$

Conclusions: In the present study, the data suggest that Fractional CO2 Laser is an effective alternative for the treatment of Minimal-Moderate Urinary Stress Incontinence in well-selected post-menopausal patients, with positive results that persist over time.

Keywords (MESH): Fractional CO2 Laser; Menopause; Urinary Incontinence
\end{abstract}

\section{INTRODUCTION}

Urinary Stress Incontinence is defined by the International Continence Society as the involuntary loss of urine, objectively demonstrable that causes a social and hygienic problem $(1,2)$. The incidence of this disease in the general population borders the $30 \%$ and $40 \%$, with its peak between 50 and 60 years. There are many factors associated with the occurrence of incontinence such as multiparity, obesity, dystopias, tobacco, constipation, as well as systemic diseases (multiple sclerosis, irritable bowel syndrome, dementia, etc.). In recent years, urinary incontinence has generated more interest in the medical and scientific community, partly due to the population aging, and the increase in life expectancy (1-4).

The pelvic floor is formed by sorts of muscular and aponeurotic structures that are attached to the bone pelvis, forming a support where the pelvic viscera are held, creating a balance of forces with the abdominal press. The muscle-fascial elements of the female perineum act together. Meanwhile, three muscles and three ligaments work as a basis for the ideal performance of urination, evacuation and continence mechanisms. Urinary continence is based on its supporting elements (5-8).

Over time, conditioning factors, such as the natural aging process, the typical hypoestrogenism of menopause, cause these structures to weaken, losing their resistance and elasticity, conditioning the appearance of urogenital dysfunctions. The pelvic floor is basically formed by the endopelvic fascia, the pelvic diaphragm, and the urogenital diaphragm, this fascia is basically fibromuscular tissue, which is responsible for suspending and stabilizing the abdominal viscera, and is mostly composed of collagen, playing a very important role in the support and balance of 
the pelvic organs, as has been demonstrated in many scientific publications (9-16).

The lower urinary tract is constituted by the bladder and the urethra, the bladder is a hollow viscus that has the function of storing urine, whose walls are formed by smooth muscle fibers, its interior is covered by the urothelium, highly specialized epithelium, that is able to be in contact with the urine, the urethra is continued, with its support system and sphincter, necessary to maintain continence. The final cause of urinary stress incontinence is the decrease in urethral pressure, which can be basically caused by two mechanisms, urethral hypermobility, because the urethral support elements are weakened or damaged, whereby, the bladder neck and the urethra descends from its normal position, when making some effort, and the intrinsic sphincter deficiency, in which there is an insufficient closure of the urethra, which could be due to an injury of the same sphincter or to a denervation of this one (17-22).

Surgeries are primarily based on correcting lax elements. Classic techniques use points for plication, which lead to the production of collagen by cicatrization. Modern techniques use polypropylene tapes to create neoligaments, producing collagen by foreign-body reaction.

On the other hand, Fractional CO2 Laser produces collagen by thermal stimulation. It is rational to assume that the production of collagen in the right place would restore ligament function, and pubocervical fascia tension.

The CO2 Laser fractionated through thermal shock proteins, stimulates collagenogenesis, at the level of the pubocervical fascia, by activating fibroblasts, creating a natural support (autologous mesh), reducing the hypermotility of this dysfunction, through a natural mechanism of cellular self-regeneration, without the possible complications of the use of meshes.

Our objective in the present study is to evaluate the effectiveness of the Fractional CO2 Laser in the conservative treatment of Urinary Stress Incontinence in pre-selected Post-Menopausal Patients, with urine loss to Valsalva maneuvers.

\section{MATERIALS AND METHODS}

Study design: A PUBA study was performed: Prospect Uncontrolled Before (the start of laser therapy) and after (the last laser therapy) in patients at the CIRUFEME Laser Female Surgery Center, Lima-Peru, between July 2015 and July 2017.

Selection of Participants: Patients who have the following conditions were included: minimum 1-year amenorrhea, no previous use of hormone replacement therapy (HRT), with involuntary loss of urine to the Valsalva maneuver, no previous pelvic floor surgeries, or surgeries with placement of meshes, genital prolapse less than grade II according to the POPQ classification, absence of active urogenital infections, smokers and with a BMI less than $26 \mathrm{~kg} / \mathrm{m} 2$.

Laser device: We use the SmartXide Touch V2LR microablative $\mathrm{CO} 2$ laser (MonaLisa Touch-Italy) with a power of $40 \mathrm{~W}$, distributed in a fluence of $6.74 \mathrm{~J} / \mathrm{cm} 2$, over the pubocervical fascia, and mid-urethral reinforcement in a second time with a fluence of $2.68 \mathrm{~J} / \mathrm{cm} 2$. The parameters that were chosen in the present study were based on the personal experience of the medical researcher.
Procedures and Methods: The patients received 3 sessions of fractional CO2 laser, with intervals of 1 month between each other. It was administered in two steps at the same time, first at the level of pubocervical fascia, and then at the level of the middle urethra, no topical anesthesia was used. After treatment, no medication was prescribed, only sexual and exercise restrictions for 1 week. The sociodemographic characteristics of the study population were collected before the start of the study, as well as the inclusion / exclusion criteria were verified before starting the first laser application. The subjective primary outcome measure was the change in the ICIQ SF validated questionnaire, as well as the application of a Visual Analogue Scale, to measure the degree of satisfaction of the treatment, whereby, in a brief and simple way, evaluate the symptoms and the impact of urinary incontinence on the quality of life of the study population.

The objective results include the application of the PAD TEST, previously weighing a female pad that was placed at the beginning of the evaluation, then indicating to drink for 15 minutes half a liter of water, and for the remaining 45 minutes he was instructed to walk, go up down stairs, and Valsalva maneuvers, finished this, the female pad was reweighted and the losses were quantified. Sample size: A total of 50 patients who met the inclusion and exclusion criteria were analyzed, all patients completed the study, reporting 1 case of urinary infection, confirmed by urine culture, which was ambulatory treated with antibiotics for 7 days, as well as two cases of vulvovaginitis, which remitted with topical ovules.

Statistical analysis: Frequencies and percentages were presented for the quantitative and median variables and interquartile ranges for the continuous variables according to the results of the normality tests (Shapiro-Wilk, meaning a value of $\mathrm{P}<0.05)$. U-mann Whitney was used for continuous non-parametric variables. The data were processed in the statistical software STATA version 14.

\section{RESULTS}

The average age of the patients was 55 years at the start of the treatment, the average onset of menopause was at 48 years, the average parity was 2 children, and the BMI overall was 23.

Of the 50 patients enrolled, 12 patients in total who presented minimal USI according to the ICIQ questionnaire, all were cured, defining this, as the no loss of urine. Of the 18 patients who presented moderate USI, 10 were cured $(55 \%)$, that is, they did not present urine loss, and the other 8 patients (45\%) had partial recovery, but continued losing urine. Of the 20 patients who presented severe USI, none were cured, 9 (45\%) of them remained in severity, and 11 (55\%) patients had partial recovery, migrating to the degree of moderate USI (Table 1).

An objective assessment was also performed using the PAD TEST, of the 12 patients with minimal USI, all were cured, of the 18 patients who presented moderate USI, what was found in the ICIQ was confirmed, with 10 patients cured, and 8 with partial recovery. Likewise, of the 20 patients with severe USI, none were cured (Table 2).

VAS (Visual Analogue Scale) was also performed at the end of the treatment and after a year by telephone call, 
to assess the degree of satisfaction, in regards to the minimal USI at the end of the treatment, this was $100 \%$, and after a year it was reduced to $95 \%$. The patients who presented moderate USI at the end of the treatment presented an average degree of satisfaction of $88.8 \%$, and after 1 year it was $72.2 \%$, and in respect of severe USI it was $6.5 \%$ at the end of the laser treatment, and after one year it was $0 \%$ (Table 3 ).

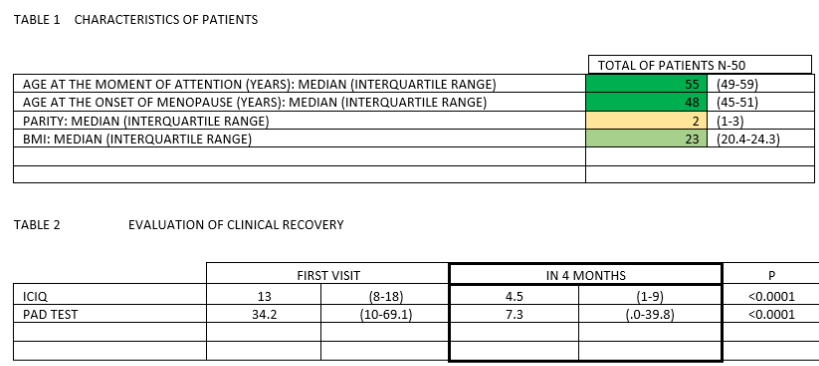

TABLE 3 GRADE OF SATISTACTION (VISUAL ANALOGUE SGAL)
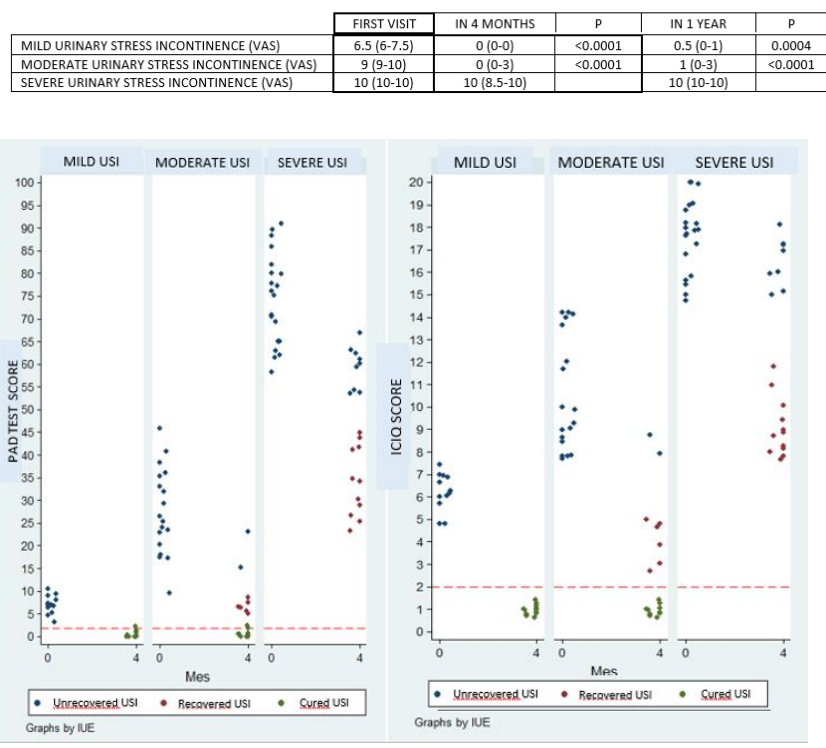

\section{DISCUSSION}

Urinary stress incontinence has a direct relationship with urethral hypermobility, and a poor tone of the pelvic musculature, due to this, the recommendations with $A$ level evidence, for the first-line treatment for USI, is rehabilitation of pelvic floor, as a conservative treatment, current trend in modern medicine, whereby, the fundamental objective of this study is to provide an option to join the existing ones, to improve the quality of life of patients, so we consider that it is very important when choosing a treatment, to identify each patient, considering the pros and cons of each option.

The treatment of USI consists basically in increasing the resistance of the urethra, so that the intravesical pressure does not exceed the urethral during efforts, the fractional $\mathrm{CO} 2$ laser by stimulating the fibroblasts, achieves this effect, by stimulating collagenogenesis, at the level of the middle urethra (Pubocervical Fascia), creating its lost support, either due to external factors, or over the years, completely reducing the adverse effects of any other invasive therapeutic option.

In the work of Debaja $\mathrm{H}$. et al, a prospective cohort study, 33 women diagnosed with SUI with urodynamic tests were evaluated, completing 3 laser sessions, analyzing their effectiveness at the end of these, through the use of sanitary towels that decreased from a median of 12 to 7 at the end of treatment, as well as questionnaires for international incontinence consultation, evidencing a significant decrease from 45 to 29.3 , and from 16 to 8.15 , only minor side effects were reported (23).

Likewise, in the study of Erel CT et al., 82 patients treated with laser were evaluated, in this cohort study, ICIQ - SF and KHQ - UI questionnaires were used before and after the procedure, at the end of treatment the mean scores of Both treatments improved significantly, it was also observed that younger women, and with normal BMI, had significantly better results, the amount of energy and the way of distributing it could also be decisive for the success of the treatment (24).

With the result of the present work, considering previously the small sample number, and may not have a statistical significance considered, we intend to demonstrate that there is a new alternative to offer to postmenopausal patients, who suffer from stress incontinence. In any case, we hope that new experiences will be published over time to strengthen the evidence regarding the use of laser in gynecology (25).

Limitations: Given the lack of randomization, placebocontrol, power analysis, and not having blinded the workers to avoid the expectation of them as a possible confusing result, the data must be interpreted with care; however, the improvement in the ICIQ-SF of the USI were highly significant in Minimal-Moderate Urinary Stress Incontinence in well-selected post-menopausal patients. Another limitation of this study would be the lack of urodynamic studies in order to detect cases of urge incontinence.

Strenghts: Some of the strengths of this study include the prospective design and the use of defined objective and subjective criteria despite the limitations. The results of this study were encouraging, with a notable perception of the effectiveness of fractionated laser treatment even after a single session in urinary incontinence.

\section{CONCLUSION}

The Fractional CO2 Laser, used in well-selected patients with ideal fluency, is an effective alternative for the treatment of minimal-moderate urinary stress incontinence, observing improvements from the beginning of the treatment, with results that last over time. This therapy should be considered as a treatment option for patients with contraindications to be operated, or simply they do not want it.

Studies In humans \& informed Consent declaration: The present study has been performed in accordance with the Code of Ethics of the World Medical Association (Declaration of Helsinki) for experiments with human beings. The institutional ethics committee of Biomedical Research Institute of Universidad Ricardo Palma in Lima, Peru, evaluated and approved the study. Informed consent was obtained for experimentation with human beings before the start of the study. 
Data Availability Statement:

https://doi.org/10.6084/m9.figshare.11955120.v1

Competing interests: The student authors are part of the educational institutions where the research was performed, however, this did not influence the results, discussion or other sections of the article.

Grant information: The authors declared that no grants were involved in supporting this work.

Funding source: Self-financed by the authors.

\section{REFERENCES}

1. Leshunov EV, Martov AG. Application of laser technologies for treatment of urinary stress incontinence in women of reproductive age. Urologiia. 2015 Jan-Feb;(1):36-40.

2. Tien YW, Hsiao SM, Lee $\mathrm{CN}$, Lin $\mathrm{HH}$. Effects of laser procedure for female urodynamic stress incontinence on pad weight, urodynamics, and sexual function. Int Urogynecol J. 2017 Mar;28(3):469-476. doi: 10.1007/s00192-016-3129-y. Epub 2016 Sep 8.

3. Hardy LA, Chang CH, Myers EM, Kennelly MJ, Fried NM. Computer simulations of thermal tissue remodeling during transvaginal and transurethral laser_treatment of female stress urinary incontinence.Lasers Surg Med. 2017 Feb;49(2):198-205. doi: 10.1002/lsm.22491. Epub 2016 Feb 22.

4. Lapii GA, Yakovleva AY, Neimark Al. Structural Reorganization of the Vaginal Mucosa in Stress Urinary Incontinence under Conditions of Er:YAG Laser Treatment. Bull Exp Biol Med. 2017 Feb;162(4):510-514. doi: 10.1007/s10517-017-3650-0. Epub 2017 Feb 27

5. Fistonić N, Fistonić I, Guštek ŠF, Turina IS, Marton I, Vižintin Z, Kažič M, Hreljac I, Perhavec T, Lukač M. Minimally invasive, non-ablative Er:YAG laser treatment of stress urinary incontinence in women--a pilot study. Lasers Med Sci. 2016 May;31(4):635-43. doi: 10.1007/s10103-016-18840 . Epub 2016 Feb 9.

6. Gaspar A, Brandi $H$. Non-ablative erbium YAG laser for the treatment of type III stress urinary incontinence (intrinsic sphincter deficiency). Lasers Med Sci. 2017 Apr;32(3):685691. doi: 10.1007/s10103-017-2170-5. Epub 2017 Feb 16.

7. Chang $\mathrm{CH}$, Myers EM, Kennelly MJ, Fried NM. Optical clearing of vaginal tissues, ex vivo, for minimally invasive laser treatment of female stress urinary incontinence. J Biomed Opt. 2017 Jan 1;22(1):18002. doi: 10.1117/1.JBO.22.1.018002.

8. Lapii GA, Yakovleva AY, Neimark AI, Lushnikova EL. Study of Proliferative Activity of Vaginal Epithelium in Women with Stress Urinary IncontinenceTreated by Er:YAG Laser. Bull Exp Biol Med. 2017 Jun;163(2):280-283. doi: 10.1007/s10517-017-3784-0. Epub 2017 Jul 18.

9. Pergialiotis V, Prodromidou A, Perrea DN, Doumouchtsis SK. A systematic review on vaginal laser therapy for treating stress urinary incontinence: Do we have enough evidence? Int Urogynecol J. 2017 Aug 2. doi: 10.1007/s00192-0173437-x

10. Arroyo C. Fractional $\mathrm{CO}$ laser treatment for vulvovaginal atrophy symptoms and vaginal rejuvenation in perimenopausal women. Int J Womens Health. 2017 Aug 28;9:591-595. doi: 10.2147/IJWH.S136857. eCollection 2017.

11. Suskind AM, Cawthon PM, Nakagawa S, Subak LL, Reinders I, Satterfield S, Cummings S, Cauley JA, Harris T, Huang AJ. Urinary Incontinence in Older Women: The Role of Body Composition and Muscle Strength: From the Health, Aging, and Body Composition Study.; Health ABC Study. J Am Geriatr Soc. 2016 Dec 5.

12. Kang D, Han J, Neuberger MM, Moy ML, Wallace SA, Alonso-Coello P, Dahm P. Transurethral radiofrequency collagen denaturation for the treatment of women with urinaryincontinence. Cochrane Database Syst Rev. 2015 Mar 18;(3). Injection devices for bulking agents in uro-gynaecology.

13. Bacsu CD, Cunningham $C$, Christie A, Zimmern PE. Durability of collagen injection for stress urinary incontinence in women proven by transvaginal 3dimensional ultrasound. Female Pelvic Med Reconstr Surg. 2015 Jan-Feb;21(1):25-9.

14. Wang L, Han LY, Li HL. Zhonghua Yi Xue Za Zhi. Etiological study of pelvic organ prolapse and stress urinary incontinence with collagen status and metabolism. $2013 \mathrm{Feb}$ 19;93(7):500-3. Chinese.

15. Chen B, Yeh J. Alterations in connective tissue metabolism in stress incontinence and prolapse. J Urol. 2011 Nov;186(5):1768-72.

16. Tunn R, Rieprich M, Kaufmann O, Gauruder-Burmester A Beyersdorff D. Morphology of the suburethral pubocervical fascia in women with stress urinary incontinence: a comparison of histologic and MRI findings. Int Urogynecol J Pelvic Floor Dysfunct. 2005 NovDec;16(6):480-6.

17. Radziszewski P, Borkowski A, Torz C, Bossowska A Gonkowski S, Majewski M. Distribution of collagen type VII in connective tissues of postmenopausal stress-incontinent women. Gynecol Endocrinol. 2005 Mar;20(3):121-6.

18. Cör A, Barbic M, Kralj B. Differences in the quantity of elastic fibres and collagen type I and type III in endopelvic fasciabetween women with stress urinary incontinence and controls. Urol Res. 2003 Jun;31(2):61-5.

19. Chen Y, DeSautel M, Anderson A, Badlani G, Kushner L. Collagen synthesis is not altered in women with stress urinary incontinence. Neurourol Urodyn. 2004;23(4):367-73.

20. Skorupski P, Rechberger T, Postawski K, Woessner JF Jr, Jakowicki JA.Is diminished pubocervical fascia collagen content a risk factor for failure of surgical management of genuine stress urinary incontinence in women? Eur J Obstet Gynecol Reprod Biol. 2002 May 10;102(2):195-8.

21. Liapis $A$, Bakas $P$, Pafiti A, Frangos-Plemenos $M$, Arnoyannaki N, Creatsas G. Changes of collagen type III in female patients with genuine stress incontinence and pelvic floor prolapse. Eur J Obstet Gynecol Reprod Biol. 2001 Jul;97(1):76-9.

22. Liapis A, Bakas $P$, Pafiti $A$, Hassiakos D, Frangos-Plemenos $M$, Creatsas $G$. Changes in the quantity of collagen type I in women with genuine stress incontinence. Urol Res. 2000 Oct;28(5):323-6.

23. Dabaja $H$, Lauterbach $R$, Matanes $E$. The safety and efficacy of $\mathrm{CO}_{2}$ laser in the treatment of stress urinary incontinence. Int Urogynecol J. 2019 Dec 18. doi: 10.1007/s00192-01904204-4.

24. Erel CT, Inan D, Mut A. Predictive factors for the efficacy of Er:YAG laser treatment ofurinary incontinence.Maturitas. 2020 Feb;132:1-6. doi: 10.1016/j.maturitas.2019.11.003. Epub 2019 Nov 9.

25. Lang $P$, Karram $M$. Lasers for pelvic floor dysfunctions: is there evidence? Curr Opin Obstet Gynecol. 2017 Oct;29(5):354-358. 